\title{
An evaluation of the availability and affordability of healthy foods in discount supermarkets
}

\author{
L. Wearing, M. McAuley and K. M. Mooney \\ Faculty of Science and Social Sciences, Liverpool Hope University, Liverpool L16 9JD, UK
}

Limited access to affordable healthy food has been proposed as a contributing factor to poor dietary choices, particularly amongst low income groups ${ }^{(1)}$. The ability to purchase healthy food has been further challenged in the United Kingdom (UK) by an increase in food prices, a phenomenon which has resulted in the market growth of discount supermarkets. The aim of this study was to compare the availability and affordability of healthy food in discount supermarkets to a non-discount supermarket (Tesco) using the Healthy Eating Indicator Shopping Basket (HEISB) ${ }^{(2)}$. This basket includes items from the five food groups based on health, choice and affordability.

The cost and nutrient composition (using the manufacturer's nutrition information panel) of the least expensive food item which met the HEISB criteria were recorded in Lidl $(n=2)$, Aldi $(n=2)$ and Tesco supermarket(s) $(n=1)$ in January 2012 . The overall nutrient quality of a range of items in the HEISB (baked beans; tinned carrots; weetabix; wholemeal loaf; yoghurt; spread) was calculated by assigning a rank of one to three to each nutrient analysed (energy; fat; saturated fat; sugar; fibre; salt) and taking the sum of the ranks. Higher levels of nutrients beneficial to health and lower levels of nutrients detrimental to health were assigned the higher rank value. The maximum possible sum of ranks was 108 .

Eighty eight percent, $94 \%$ and $97 \%$ of the food items in the HEISB were available in Lidl, Aldi and Tesco, respectively. Aldi and Tesco stocked all fruit and vegetable items. Tesco also stocked all carbohydrate rich items, where as Lidl and Aldi stocked seven and eight items from this food group, respectively. All three supermarkets stocked the same number of items from the milk and dairy group $(n=3)$ and the protein rich group $(n=3)$. The cost of the HEISB was highest in Tesco (Lidl: £41.82; Aldi £44.80; Tesco £55.26) and remained the highest when food items not available in all three supermarkets were excluded from analysis. The overall nutrient quality of the selected items in the basket was similar amongst the three supermarkets (figure 1).

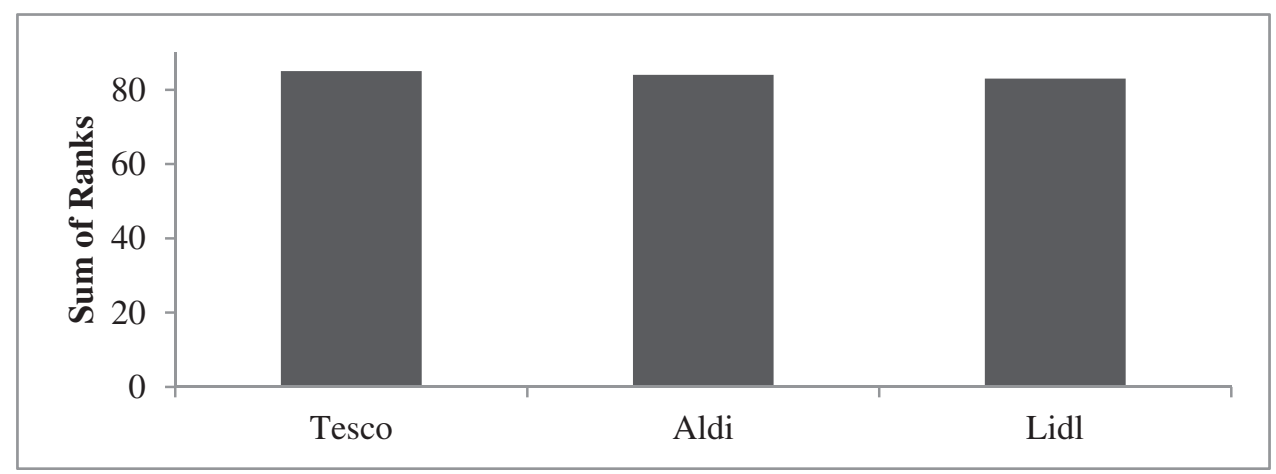

Figure 1. Sum of ranks of the nutrient content of selected foods from the HEISB.

Overall these results suggest that discount supermarkets provide access to a wide range of healthy foods from the five food groups at a lower price when compared to the dominant supermarket retailer in the UK.

1. Reisig V \& Hobbiss A (2000) Health Education Journal 59, 137-149.

2. Dawson et al. (2007) Accessing Healthy Food: A Sentinel Mapping Study of Healthy Food Retailing in Scotland. Food Standards Agency Scotland; Research Project S04005. 